\title{
Considerações metodológicas sobre o estudo de caso na pesquisa em psicoterapia'
}

\author{
Methodological considerations concerning \\ case studies in psychotherapy research
}

\author{
Fernanda Barcellos SERRALTA2 \\ Maria Lúcia Tiellet NUNES \\ Cláudio Laks EIZIRIK ${ }^{4}$
}

\begin{abstract}
Resumo
O presente artigo faz uma revisão crítica da literatura acerca da aplicabilidade do estudo de caso na pesquisa em psicoterapia: a) apresenta as características do estudo de caso como estratégia de pesquisa; b) aborda a importância dos delineamentos de caso único no estudo do processo terapêutico; c) discute estatégias para aumentar a fidedignidade e validade dos estudos de caso; d) apresenta exemplos de estudos de caso oriundos da atual geração de pesquisa empírica em psicoterapia e psicanálise. De modo geral, este estudo destaca o potencial dos delineamentos intensivos e sistemáticos de caso único, para a investigação dos processos subjetivos e complexos inerentes ao encontro terapêutico.
\end{abstract}

Unitermos: Estudos de casos. Metodologia. Psicoterapia.

\begin{abstract}
The article reviews the literature concerning the applicability of case studies in psychotherapy research: a) it presents the characteristics of case studies as a research strategy, b) addresses the importance of single-case designs in the study of the therapeutic process, c) discusses strategies to increase the reliability and validity of case studies, and d) provides examples of case studies from the current generation of empirical research in psychotherapy and psychoanalysis. In general, the article highlights the potential of single-case intensive and systematic delineations for research into the subjective and complex processes inherent to therapeutic encounters.
\end{abstract}

Uniterms: Case studies. Mathodology. Psycotherapy.

A pesquisa em psicoterapia e em psicanálise ${ }^{\mathbf{5}}$ pressupõe a aplicação de métodos empíricos aos dados clínicos, para responder a duas questões centrais: quais as mudanças que ocorrem durante a terapia e em consequência dela? como essas mudanças ocorrem? A primeira questão refere-se ao resultado, e a segunda, ao

\section{$\boldsymbol{\nabla \nabla \nabla \nabla}$}

- Artigo elaborado a partir da tese de F.B. SERRALTA, intitulada "A relação entre processo e resultado na psicoterapia psicodinâmica breve: um estudo de caso". Universidade Federal do Rio Grande do Sul, 2010.

2 Universidade Luterana do Brasil, Curso de Psicologia. Av. Farroupilha, 8001, São José, 92425-900, Canoas, RS, Brasil. Correspondencia para/Correspondence to: F.B. SERRALTA. E-mail: <psifer@terra.com.br>.

3 Pontifícia Universidade Católica do Rio Grande do Sul, Faculdade de Psicologia, Pós-Graduação em Psicologia. Porto Alegre, RS, Brasil.

- Universidade Federal do Rio Grande do Sul, Departamento de Psiquiatria e Medicina Legal. Porto Alegre, RS, Brasil.

5 O conceito de pesquisa aqui empregado refere-se à aplicação de métodos derivados de outras ciências, ao estudo dos dados oriundos da clínica psicanalítica. Propositalmente, ao longo deste artigo não se abordou a pesquisa psicanalítica no sentido proposto por Freud, ou seja, aquela que é realizada com o método psicanalítico durante o processo analítico. 
processo terapêutico. Embora teoricamente processo e resultados sejam interligados, na prática eles se referem a diferentes tipos de pesquisa, sendo que "os métodos que fornecem as melhores avaliações em uma área, muitas vezes, são em termos operacionais contrários àqueles que fornecem as melhores avaliações na outra" (Wallerstein, 2007, p.305).

A evolução histórica da pesquisa em psicoterapia atesta que a avaliação de resultados e o interesse nas diferenças entre grupos dominou o cenário nas décadas de 1970 e 1980 (Araújo \& Wiethaeuper, 2003; Serralta \& Streb, 2003; Wallerstein, 2002; 2007). Esses estudos, embora fundamentais para estabelecer critérios de tratamento baseado em evidências e assim subsidiar decisões em políticas de saúde (Levy \& Ablon, 2009), via de regra exercem pouca ou nenhuma influência sobre a teorização e a prática clínica, uma vez que geralmente excluem o exame de variáveis mais complexas (como a natureza da relação terapêutica, por exemplo) (Jones, 1993). A teorização clínica pressupõe um exame minucioso do encontro terapêutico e suas múltiplas facetas. Informações essenciais para esta compreensão são obviamente perdidas nos estudos grupais (Hilliard, 1993; Levy \& Ablon, 2000).

De modo geral, psicoterapeutas e psicanalistas consideram que a pesquisa é reducionista, simplista e dissociada da realidade do consultório. De fato, para subsidiar a prática clínica, é necessário o desenvolvimento de estudos que focalizem as variações intrassujeitos e as evidências empíricas dos mecanismos de mudança em psicoterapia (Pole, Ablon, O'Connor \& Weiss, 2002). Esse é o alvo dos estudos de processo. Estudar processo implica examinar variáveis complexas, multifacetadas e contextualizadas; supõe, portanto, avaliação do que ocorre durante o tratamento (Kazdin, 2001). Por conseguinte, o estudo de caso assume aqui uma posição central.

Muitos pesquisadores são reticentes quanto ao estudo de caso como estratégia de pesquisa válida. Confusões conceituais, falsas generalizações e mitos sobre as limitações dos delineamentos de caso único ou múltiplos permeiam a literatura. Mal compreendido por muito tempo, esse tipo de estudo foi visto, erroneamente, como tendo validade científica questionável (Hilliard, 1993; Howard, 1993). Um olhar mais atento

502 revela, entretanto, que muito do ceticismo acerca do valor do estudo de caso como estratégia de pesquisa efetiva se deve "à falta de compreensão da lógica básica da pesquisa de caso único" (Jones, 1993, p.371). Um problema adicional, conforme Alves-Mazzotti (2006), é o falso pressuposto de que estudos de caso são fáceis de realizar e por isso recomendáveis aos investigadores iniciantes. Afirmativas como esta desconsideram a complexidade inerente a esse tipo de pesquisa e contribuem para que muitos trabalhos sejam erroneamente considerados "estudos de caso".

Mas, afinal, o que constitui um estudo de caso como estratégia de pesquisa no campo das psicoterapias? Em primeiro lugar, é relevante delimitar uma diferenciação entre estudos ou relatos de casos baseados no método clínico dos estudos de caso delineados como estratégia de pesquisa empírica.

Tradicionalmente, o relato de casos tem sido a base de pesquisa nos estudos sobre processo da psicanálise e psicoterapia psicanalítica. E, como salienta Bucci (2007), do ponto de vista de muitos analistas, esse método ainda hoje é considerado necessário e suficiente para produzir conhecimento.

Por outro lado, nas últimas décadas, o crescente reconhecimento dos problemas clínicos e científicos associados ao relato de casos (como, por exemplo, a tendenciosidade do observador/relator, a omissão ou perda de dados, e os efeitos da intenção de estudar o caso sobre o tratamento) abriu caminho para o estudo sistemático de casos únicos ou múltiplos, realizado através de medidas objetivas aplicadas a sessões gravadas de psicoterapia ou análise.

Como clínicos e pesquisadores, os autores deste artigo compartilham do ponto de vista de que ambas as perspectivas, a que defende a utilização do método psicanalítico propriamente dito e a que incorpora a aplicação de métodos empíricos para o estudo de tratamentos psicanalíticos, são igualmente válidas e necessárias para a produção do conhecimento, sendo esta última particularmente importante para ampliar a interlocução entre psicanálise e demais campos de saber.

Neste artigo, focaliza-se intencionalmente o estudo de caso em sentido mais estrito, por se acreditar que é justamente esse tipo de estudo o que possui maior potencial para diminuir o hiato considerável que existe entre a pesquisa empírica e a prática clínica. Os autores estão bastante cientes das dificuldades de men- 
surar, avaliar e submeter à prova empírica processos altamente complexos e intersubjetivos, como os que compõem o encontro paciente-terapeuta. Entretanto, compartilham o ponto de vista de que é possível e necessário que a psicanálise e as psicoterapias se submetam a exame científico, não apenas a partir de sua própria perspectiva, mas também desde a perspectiva das demais ciências. A experiência dos autores revela que o estudo de caso é um delineamento que favorece essa integração, especialmente quando conduzido de modo a contemplar o controle dos seus possíveis vieses.

O objetivo desta revisão, portanto, é apresentar e discutir os alcances e limitações do estudo de caso como estratégia de pesquisa empírica em psicoterapia e psicanálise. Neste sentido, o presente trabalho apresenta também algumas formas de incrementar o rigor metodológico dos estudos realizados neste âmbito. Acredita-se que isso poderá auxiliar clínicos e pesquisadores a refinar seus métodos de investigação, conjugando subjetividade e objetividade, de modo a contemplar exigências e interesses clínicos e metodológicos.

\section{Método}

Foi realizada uma revisão crítica e não sistemática da literatura sobre o assunto, nas bases de dados Lilacs, Index-Psi, MedLine e PsychInfo, com os seguintes descritores: relato de caso (case report), estudo de caso (case study) estudos de caso (case studies) ou caso único (single case), sem limitação de data. A busca foi então combinada com os termos psicoterapia (psychotherapy) ou psicanálise (psychoanalysis) e pesquisa (research). Foram excluídos artigos que não abordassem as funções, aplicabilidade e características do estudo de caso com possíveis implicações para a pesquisa em psicoterapia. Complementou a revisão uma busca, em bibliotecas universitárias locais, por manuais de pesquisa em ciências humanas, ciências médicas e ciências sociais.

O material bibliográfico revisado foi organizado em 4 tópicos centrais: o estudo de caso como elemento de ligação entre a pesquisa empírica e a prática clínica; caracterização do estudo de caso como estratégia de pesquisa; considerações sobre as inferências nos estudos de caso; e dois exemplos de estudos de caso empírico.

\section{O estudo de caso como elemento de ligação entre a pesquisa empírica e prática clínica}

Existe uma dissociação histórica entre pesquisa e atividade psicoterápica, e entre pesquisa e psicanálise: enquanto os clínicos exploram os complexos domínios da subjetividade humana, os pesquisadores têm priorizado a avaliação de processos e resultados terapêuticos, através de delineamentos experimentais de tratamentos manualizados que não refletem nem representam a prática clínica convencional (Drozd \& Goldfried, 1996; Neufeldt \& Nelson, 1998). Tipicamente, psicoterapeutas e psicanalistas exercem suas atividades longe dos centros acadêmicos e de pesquisa, e exibem um nítido desinteresse pelas ciências empíricas (Lancelle, 1997). Sua formação não inclui treinamento em metodologias não-psicanalíticas nem o estudo de outras áreas do conhecimento (Kandel, 2003; Kernberg, 2002). Essa pobre interlocução entre a psicanálise e as demais ciências reforça a falsa crença, amplamente difundida, de que as capacidades clínica e científica são antagônicas e incompatíveis (Westen, 2002).

Analisando a literatura sobre pesquisa e psicoterapia, é difícil discordar da afirmativa de Gabbard (2009), segundo a qual, em termos científicos, os tratamentos psicodinâmicos são o "primo pobre" de outros tratamentos muito mais estudados por métodos empíricos (como é o caso das psicoterapias cognitivo-comportamentais e dos tratamentos psicofarmacológicos, por exemplo). Uma revisão da literatura sobre pesquisa psicanalítica na ISI Web ofScience feita por Weiss (2009) revelou que entre 1997 e 2006 os termos "psicodinâmica" (ou "psicoterapia psicodinâmica") e "pesquisa" geraram ínfimos 226 resultados, sendo ainda observado que a taxa de crescimento de produção científica no período aumentou apenas 1\%, quando o crescimento geral desse tipo de produção ficou entre 5\% e 15\%. Por outro lado, o exame da literatura revela que atualmente existe um movimento crescente no sentido de desenvolver subsídios para uma psicoterapia psicanalítica baseada em evidências e para aumentar a compreensão sobre seus mecanismos de ação terapêutica (Gabbard, 2009).

Sabe-se, entretanto, que a adoção de métodos derivados das demais ciências (e portanto, não-psicanalíticos) para o estudo da clínica psicanalítica, é polêmica 
e controversa ${ }^{6}$. Luyten, Blatt e Corveleyn (2006) identificam duas posições contrárias: a dos que defendem a tradição fundamentalmente interpretativa das narrativas de casos psicanalíticos usados por Freud para a construção da teoria (geralmente autores de língua francesa); e a dos que adotam uma posição "neopositivista" e preconizam a adoção de modelos estatísticos, experimentais e/ou quase-experimentais para estudar relações de causa e efeito e explicar dados oriundos da clínica psicanalítica (via de regra, autores de língua inglesa).

Entretanto, adotar métodos empíricos para o estudo de dados clínicos não significa necessariamente abdicar da tradição psicanalítica e ingressar no universo das hard sciences. A complexidade, subjetividade e multidirecionalidade do processo terapêutico pressupõe o desenvolvimento e a utilização de metodologias compatíveis com as questões propostas por esse campo de pesquisa. E pque isso ocorra, é necessário suplantar a discussão sobre as possibilidades de avaliar processos subjetivos através de métodos empíricos e sair da zona de conforto, substituindo o debate pela ação.

Nesse sentido, a literatura exibe um verdadeiro chamado para o desenvolvimento de uma "ciência da subjetividade" (Kächele, Schachter \& Thomä, 2007), ou seja, para um refinamento metodológico dos tradicionais estudos de casos clínicos, gerando assim uma maior integração entre as abordagens clínica e empírica, idiográfica e nomotética. Esse movimento é ainda muito pequeno no Brasil, onde raríssimos são os trabalhos que buscam compreender o processo terapêutico a partir de métodos que aliem validade clínica e empírica. É necessário, portanto, incentivar a produção científica nacional nesse campo.

\section{Estudo de caso como estratégia de pesquisa}

Com base na revisão da literatura realizada, pode-se entender o estudo de caso como um subtipo de pesquisa de caso único, que constitui uma estratégia de investigação naturalística e flexível, mediante a utilização de múltiplos métodos (quantitativos e/ou qualita- tivos) e variadas fontes de evidências e informações, para descrever de forma intensiva e profunda um ou mais casos individuais (seja o paciente, a díade paciente-terapeuta, o processo terapêutico etc.), focalizando as variações intrassujeitos através do tempo, a fim de testar proposições teóricas (hipóteses) ou gerar explicações a serem testadas e/ou confirmadas em estudos ulteriores. Diferenciam-se, portanto, estudos de caso empíricos e estudos de caso clínicos. Essas características são apresentadas e comentadas a seguir:

O estudo de caso é uma pesquisa observacional naturalística de caso único: nas pesquisas de caso único em psicoterapia, distinguem-se os modelos experimentais e os observacionais (Hilliard, 1993). Os delineamentos experimentais e quase-experimentais são bastante restritivos: exigem, entre outros aspectos, medidas bem definidas dos comportamentos que devem ser modificados pelo tratamento (resultados) e que sejam reativas à intervenção. Esses delineamentos também pressupõem que os comportamentos-alvo sejam reversíveis na retirada do tratamento (Backman \& Harris, 1999; Silvares, 2004). Assim, embora muitas vezes os delineamentos de caso único sejam genericamente denominados de estudo de caso, em sentido mais estrito reserva-se o termo para os estudos não experimentais, diferenciando-os desse modo dos experimentos de caso único (Hilliard, 1993).

O estudo de caso é uma estratégia de pesquisa pluralista do ponto de vista metodológico: o estudo de caso é uma estratégia de pesquisa pluralista (Denscombe, 2003; Hilliard 1993; Stake, 2003; Yin, 2001). Desse modo, embora seja uma das formas mais comuns e conhecidas de pesquisa qualitativa, o estudo de caso não é, em sua essência, uma metodologia qualitativa. A sua inclusão entre as metodologias qualitativas possivelmente reflete a falsa equiparação entre qualitativo e idiográfico (Hilliard, 1993), bem como uma confusão entre método de coleta de dados e estratégia de pesquisa (Yin, 1981). Estudos de caso único (ou múltiplos) podem envolver dados qualitativos (geralmente expressos em forma de prosa ou narrativa), bem como dados quantitativos (mensurações em nível nominal, ordinal, intervalar ou de razão, submetidas a procedimentos de estatística

$\operatorname{rove}$

- Uma das controvérsias mais conhecidas é a existente entre Robert Wallerstein e André Green, cujos pontos de vista contrastantes foram publicados no 504 newsletter da Internacional Psychoanalytical Association (IPA), em 1996. 
descritiva e/ou inferencial); também podem ser híbridos, isto é, conjugar as duas abordagens (Stake, 2003; Yin, 2001).

Um caso é um fenômeno individual que étomado como unidade de análise e interesse: nos estudos de caso, o caso é um fenômeno individual, particular, complexo e único (Denscombe, 2003). As pesquisas de caso único em psicoterapia são aquelas que consideram uma entidade individual (paciente, díade terapeuta-paciente, terapia de grupo, ou interação entre esses) como unidade de análise e de interesse (Eells, 2007). O que define o caso, portanto, é o fato de ele constituir uma unidade específica, um sistema cujas partes estão integradas (Stake, 2003).

Um estudo de caso envolve descrições em profundidade: Estudos de caso são estudos intensivos, nos quais o pesquisador está interessado em obter uma grande quantidade de dados, em um número limitado de participantes. Diferem das investigações de grupo nas quais um número limitado de dados é colhido e analisado em um grande número de sujeitos (Chassan, 1979). Estudos de caso realizam descrições cuidadosas, detalhadas e aprofundadas, que podem ser realizadas através de variadas técnicas de avaliação ou mensuração (Backman \& Harris, 1999).

Oestudo de caso éuma estratégia de pesquisacom planejamento flexível: a adaptatividade e a flexibilidade são outras características dessa estratégia de pesquisa. Conforme Yin (2001), "pouquíssimos estudos de caso terminarão exatamente como foram planejados" (p.83). Isso significa que esse tipo de investigação permite ao pesquisador, em virtude das necessidades que emergem durante o próprio estudo, alterar a direção prevista ou incluir outro caso, por exemplo. A possibilidade de desvio do planejamento representa um dos pontos mais fortes do estudo de caso, embora isso potencialmente possa tornar o estudo mais trabalhoso e exigir do pesquisador capacidade de adaptação e habilidade em manter o equilíbrio entre flexibilidade e rigor (Robson, 1993).

Um estudo de caso avalia processos inter-relacionados numa perspectiva temporal: estudos de caso tipicamente focalizam processos que são inter-relacionados. Como tendem a observar e descrever o caso como um todo, são passíveis de explicar como as partes se conectam umas com as outras (Denscombe, 2003).
As questões de pesquisa geralmente referem-se ao como e ao por que (Yin, 2001) e focalizam relações complexas (Stake, 2003). Em psicoterapia, os estudos de caso possibilitam a análise longitudinal da imbricada interação entre as diversas variáveis da dimensão processos-resultados (Hilliard, 1993).

O estudo de caso pode ser usado em ambos os contextos: de descoberta e de justificativa : a visão comum do estudo de caso como um estudo piloto, apropriado às etapas preliminares de investigação, é simplista e equivocada (Flyvbjerg, 2006). Estudos de caso podem ser explanatórios, descritivos ou exploratórios (Yin, 2001): quando o estudo envolve o teste de hipóteses formuladas a priori, o contexto é de justificativa e o estudo é confirmatório (ou explanatório); quando o objetivo é gerar hipóteses para serem testadas futuramente, o contexto é de descoberta e o estudo é exploratório (Hilliard, 1993).

Oestudo de caso empírico é diferente do estudo de caso clínico em termos de objetivos e métodos: desde os primóridos da clínica psicanalítica, as monografias, relatos e vinhetas clínicas são frequentemente realizadas com o objetivo de descrever, informar, compartilhar experiências e ilustrar teorias e técnicas, demonstrando a plausabilidade das mesmas (d'Allones, 1989; Aveline, Strauss \& Stiles, 2007; Widlocher, 1994). Nesse sentido, o estudo de caso pode ser considerado "a matéria prima por excelência da psicanálise", a origem dos principais insights até o momento obtidos (Eizirik, 2006, p.172). Entretanto, via de regra, os estudos clínicos, ao contrário dos estudos empíricos, são conduzidos sem qualquer procupação com o rigor metodológico (Widlöcher, 1994). Isso faz com que pesquisadores os considerem muito limitados como forma de pesquisa (Bucci, 2007; Fonagy, 2004; Messer, 2007). Messer (2007) lista algumas dessas limitações: a narrativa baseia-se na memória do terapeuta ou analista e pode, portanto, ser afetada por desejos, temores, expectativas e outros fenômenos inconscientes; a escolha do material analisado é sem nenhum controle de viés de seleção; a interpretação do material segue a ortodoxia teórica dominante e ignora outras possibilidades de compreensão do fenômeno; há poucos dados contextuais que permitam ao leitor confirmar ou refutar hipóteses sobre o significado do material; não há medidas de resultados, além da impressão subjetiva do terapeuta ou analista. 
Em resposta a essas restrições, nas últimas décadas, enormes esforços têm sido despendidos para refinar instrumentos e métodos aplicáveis ao estudo de caso individual. A progressiva aceitação, por parte dos psicanalistas e psicoterapeutas, dos procedimentos de gravação das sessões e o desenvolvimento de novas tecnologias de coleta e análise de dados inauguram um novo estágio na pesquisa clínica psicanalítica (Bucci, 2007; Kächele \&Thomä, 2007; Serralta \& Streb, 2003; Wallerstein, 2002; 2007). Esse tipo de estudo (denominado na literatura de estudo de caso sistemático, empírico ou quantitativo, conforme o caso) compartilha com os estudos clínicos tradicionais a abordagem idiográfica e o estudo contextualizado do fenômeno, porém pressupõe o exame empírico dos achados clínicos, o que requer cuidados metodológicos que assegurem a validade interna e externa do estudo (Kächele, Schachter \&Thomä, 2007).

\section{Considerações sobre as inferências nos estudos de caso}

A forma mais usualmente praticada para aumentar a confiabilidade de dados em estudos de caso é conhecida como triangulação. A triangulação pode ser de dados (múltiplas fontes de informação), de pesquisadores (diferentes avaliadores), de metodologia (uso de diferentes métodos) e de teorias (uso de diferentes perspectivas teóricas para compreender o mesmo conjunto de dados) (Patton, 1987).

Estudos de caso, assim como outras estratégias de pesquisa, buscam desenvolver teorias passíveis de verificação empírica. É importante compreender que a lógica que sustenta a generalização dos dados nos estudos de caso é distinta da lógica dos estudos amostrais. No estudo de caso, o pesquisador possui o objetivo de "expandir e generalizar teorias (generalização analítica) e não enumerar freqüências (generalização estatística)"(Yin, 2001, p.29). Isso significa que a relação proposta entre duas ou mais características encontradas não está baseada na representatividade da amostra, mas sim em um nexo causal plausível entre essas características (Mitchell, 1983).

Desse modo, as características do estudo de caso se estendem a outros casos, pela força do raciocínio explicativo (Yacuzzi, 2005). A falta de compreensão dessa lógica leva à crítica frequente de que não é possível generalizar a partir de estudos de caso. A capacidade de generalização pode ser aumentada através da escolha estratégica do caso em estudo. Em determinados contextos, por exemplo quando se quer extrair a maior quantidade possível de informação sobre determinado fenômeno, pode ser mais útil a seleção de um caso extremo ou atípico do que um caso típico ou representativo da população-alvo. Estudos de caso são ainda particularmente adequados para generalizar a partir do que Karl Popper denominou falsificação, ou seja, para falsear hipóteses a partir de observações profundas e, portanto, mais passíveis de encontrar diferenças sutis não perceptíveis nos estudos grupais (Flyvbjerg, 2006).

A validade interna nos estudos explicativos refere-se à veracidade das inferências extraídas da análise do caso. Entre as ferramentas mais utilizadas para assegurar esse tipo de validade estão: o exame do comportamento das variáveis em estudo em relação a um padrão previamente definido (através de outros estudos, por exemplo); a construção de explanações ou de elos causais em relação ao evento ou fenômeno em estudo; e as análises cronológicas dos acontecimentos e de séries temporais (análogas às usadas em experimentos com grupos), que buscam examinar como e por que se dão as relações dos eventos ao longo do tempo (Yin, 2001).

Contrariamente ao que ocorre nos delineamentos grupais, o acréscimo de novos casos não necessariamente aumenta a confiabilidade dos dados. Nesse tipo de delineamento, o acréscimo inteligente de novos casos só deveria acontecer após o fenômeno em estudo estar suficientemente compreendido no nível individual. A inclusão prematura de um novo caso pode ser extremamente nefasta à compreensão do caso em estudo (Hilliard, 1993). Tendo em consideração essa ressalva, a replicação é a chave para a generalização dos dados (validade externa), e também para incrementar a fidedignidade dos achados (validade interna) oriundos de estudos de caso (Galassi \& Gersh, 1993).

\section{Exemplos de estudos de caso empírico}

Foram selecionados, na literatura, dois exemplos de estudos de caso único produzidos por pesquisadores da atual geração de pesquisa em psicoterapia: um caso 
de psicanálise e um de psicoterapia psicanalítica. A escolha dos casos foi intencional e baseou-se em sua capacidade de representar uma pequena amostra do que atualmente está sendo realizado nesse contexto. Nos exemplos, este estudo procurou ater-se mais ao método e planejamento da pesquisa do que às conclusões acerca das hipóteses clínicas.

O primeiro exemplo, o caso Amália X, é o caso prototípico do modelo de pesquisa clínica e empírica proposto pelo grupo de UIm (Kächele, Schachter \& Thomä, 2007). A paciente, uma mulher de 30 anos, buscou tratamento psicanalítico devido à baixa autoestima e à depressão neurótica relacionada ao hirsutismo (crescimento excessivo de pelos em áreas anatômicas características de distribuição masculina). O tratamento de Amália teve um total de 517 sessões, todas gravadas em áudio.

O caso Amália foi estudado por diversos métodos e instrumentos em numerosos estudos.

Relata-se aqui o estudo de Albani, Pokorny, Blaser, Geyer e Kächele (2007), que aplicou pela primeira vez o método Core Conflictual Relationship Theme (CCRT) de Luborsky em um caso de psicanálise. Esse método visa à descrição de padrões internalizados de relacionamento. No estudo, foi utilizada uma amostra das primeiras 25 e das últimas 25 sessões, caracterizando as fases inicial e final do tratamento. Adcionalmente, começando com a 50a sessão, foram avaliados blocos de 5 sessões a cada 50 sessões de intervalo. As avaliações foram realizadas por um avaliador experiente. Para verificar a fidedignidade dessas avaliações, 1 a cada 11 sessões foram aleatoriamente selecionadas para serem avaliadas por um segundo avaliador. Foram então examinados os índices de acordo entre os avaliadores, através do coeficiente Kappa.

Em uma tabela bidimensional de contingência, a variável "fase da terapia" foi cruzada com as variáveis do CCRT, desejos, necessidades e intenções (componente $W$ ), reações do objeto (componente $\mathrm{RO}$ ) e reações do self(componente RS). A hipótese dos pesquisadores foi a de que a frequência das categorias do CCRT variavam conforme a fase de tratamento.

Nas 92 sessões das 22 fases avaliadas, foram encontrados 579 episódios de relacionamento, contendo 806 desejos, 986 reações do objeto e 1103 reações do sujeito. Pelo método CCRT, os temas centrais de conflito nos relacionamentos, mais frequentes para a análise como um todo, foram: os outros devem me dar atenção (WO); eu quero ser autodeterminada (WS); os outros não-confiáveis (RO); estou insatisfeita, com medo (RS).

As categorias "mais frequentes do que o esperado" caracterizam os temas que distinguem uma fase da outra. Por exemplo, o início da análise (fase 1), caracteriza-se pelo desejo de receber atenção dos outros (WO). Amália queixa-se de se sentir "usada" por seus colegas $(\mathrm{RO})$, com quem não consegue dividir seus problemas. Sente inveja das colegas. Também se sente insegura perante suas alunas (RS), pensando que estas a consideram como uma velha empregada (RO). Existem conflitos nos quais não se sente apoiada por seu diretor (RO). Descreve seu pai como uma pessoa assustadora e inacessível $(\mathrm{RO})$ e sente-se chateada pelo relacionamento distante e conturbado com ele (WO). Já no término da análise (fases 21 e 22), Amália preocupa-se predominantemente com as experiências relacionadas a seu último relacionamento amoroso e com o inicio de um novo relacionamento (WO). Uma reunião com sua arqui-inimiga gera sentimentos intensos de ódio, que ela consegue administrar (WS). Na esfera profissional, ela é capaz de fazer valer a sua vontade (WS) e sente orgulho disso (RS). A conclusão da análise e a separação da analista são temas centrais dessa fase.

O estudo do caso Amália X demonstrou que as diferentes fases da análise podem ser caracterizadas por "conflitos focais", que podem ser operacionalizados pelo método CCRT. Em contraste com a subjetividade das descrições clínicas, tipicamente realizadas por linguagem metafórica, o CCRT permite uma abordagem diferenciada e menos subjetiva dos temas que emergem durante o processo de análise.

O segundo exemplo é derivado do modelo proposto pelo Berkeley Research Group, cuja proposta denominada "análise quantitativa de caso único" gerou, desde o início da década passada, uma série de estudos nos quais são aplicados procedimentos de análise de séries temporais, para compreender o curso da psicoterapia ou da análise (Jones, 2001). No primeiro estudo dessa série, Jones, Graham, Nigg e Dyer (1993)apresentam o que constitui, segundo eles, um novo paradigma na pesquisa de caso único neste campo. 
O caso em estudo foi uma psicoterapia psicodinâmica, com duração de dois anos e meio, duas vezes por semana. A paciente foi uma mulher deprimida de 35 anos. A terapeuta foi uma psicóloga clínica bastante experiente. O protocolo da pesquisa incluiu a aplicação de medidas de resultado terapêutico no início do tratamento e a cada 16 sessões. As medidas incluíram instrumentos autorrespondidos pela paciente e outros pela terapeuta, compreendendo avaliação diagnóstica, de sintomatologia, de personalidade, de ajustamento social, de problemas interpessoais e de pensamentos negativos. A avaliação do processo terapêutico foi realizada através do Psychotherapy Process Q-set (PQS), intrumento aplicado por juízes independentes treinados, e que envolve o estudo intensivo das sessões gravadas e transcritas do tratamento. Após a certificação de boa fidedignidade entre os avaliadores, foram ordenados os elementos do processo terapêutico (estados mentais do paciente, relacionamento terapêutico e técnica do terapeuta) mais e menos característicos. Isso permitiu a obtenção de uma descrição geral do processo. Posteriormente, procedimentos de análise fatorial exploratória foram aplicados para identificar as dimensões do processo.

Quatro fatores foram encontrados. O primeiro fator, denominado "Aceitação/Neutralidade do Terapeuta", captou a postura empática, de aceitação incondicional, facilitadora, assim como a percepção acurada do processo por parte da terapeuta. O segundo fator, "Interação da Terapeuta", foi composto por itens que descrevem um padrão de transferência-contratransferência, no qual a terapeuta controla a interação, tem respostas emocionais e pessoais à paciente, torna-se autoritária ou didática, enquanto a paciente tem dificuldades de entender a terapeuta e se sente pouco compreendida por ela. O terceiro fator, correspondente à "Técnica Psicodinâmica", incluiu o uso de interpretações de desejos e ideias inconscientes, a ênfase em sentimentos que a paciente acha intoleráveis, a interpretação de defesas e o uso da clarificação, entre outras técnicas. O quarto e último fator, "Afetos Disfóricos da Paciente", agregou itens que descrevem os afetos negativos da paciente e sua tentativa de controlá-los na sessão. Esses quatro fatores foram utilizados como variáveis na análise de séries temporais, que buscou verificar a relação de influência causal entre cada um deles e as medidas repetidas de resultados, e entre cada um dos elementos do processo entre si. Finalmente, foi testada a hipótese do efeito das técnicas de apoio versus técnicas de insight, sobre a evolução dos sintomas da paciente.

De modo geral, os resultados indicam que paciente e terapeuta exercem influência mútua, uma influenciando a outra. Por exemplo, os afetos disfóricos da paciente (fator 4) predizem a aceitação e neutralidade da terapeuta (fator 1) e este fator prediz o uso de técnicas psicodinâmicas (fator 3). A técnica psicodinâmica (fator 3) e os afetos disfóricos da paciente (fator 4) mostraram relação complexa, bidirecional: o fator 3 determina o fator 4 e vice-versa. A análise das técnicas expressivas versus técnicas de apoio também mostrou resultados complexos e indica que a terapeuta, influenciada pela paciente, varia as suas técnicas e que ambas as técnicas, de apoio e de insight, contribuem para a melhora da paciente. Esse estudo apresenta, portanto, um modelo de avaliação do processo terapêutico e da relação entre processo e resultados que é multivariado, capaz de avaliar processos de influência múltua e recíproca, em uma perspectiva quantitativa longitudinal.

\section{Considerações Finais}

Um dos maiores desafios colocados aos pesquisadores em psicoterapia é o de aumentar a relevância clínica dos estudos da área. Na pesquisa psicanalítica, esse desafio é um imperativo, pois para ser psicanalítica e científica (no sentido mais estrito do termo), a investigação necessita conjugar a alta complexidade e a subjetividade da interação terapêutica com as exigências de objetividade das ciências empíricas. Com base na literatura, o presente artigo defende que essa tarefaé facilitada pela adoção do estudo de caso como estratégia de pesquisa. Trata-se de uma ferramenta poderosa: com relativamente pouca intrusão na condução do tratamento, pode ser utilizado, com a adição de alguns refinamentos metodológicos, para estudar dados naturalísticos, oriundos da atividade clínica usual - e não de situações artificiais -, de modo a contemplar tanto os requisitos da ciência como os da prática clínica.

Uma das maiores vantagens dos estudos de caso único é sua ampla aplicabilidade: servem para gerar e para testar hipóteses; podem servir à investigação do processo terapêutico e também à de processo-resul- 
tado; podem restringir-se a um caso ou ser extensivos a múltipos casos; podem ser úteis a diversas modalidades de intervenção clínica. Nos estudos que examinam os complexos mecanismos de mudança em psicoterapia, o estudo de caso é considerado o método de excelência.

Estudos de caso também possuem limites, entre os quais se destacam a validade interna e a externa. No estudo das psicoterapias não comportamentais, os desafios parecem ser ainda maiores. Entretanto, ainda que as questões de validade interna e externa representem um constante desafio aos pesquisadores, existem meios de diminuir as ameaças à validade dos estudos de caso em psicoterapia, com a adoção de medidas que visem a aumentar o rigor e a capacidade de generalização dos estudos realizados. Isso tem ocorrido nos estudos sistemáticos de caso. Esses, embora compartilhem com os estudos qualitativos tradicionais muitas das suas características, geralmente exibem maior refinamento metodológico (por exemplo, utilizando material clínico gravado em vídeo e/ou áudio, avaliando os dados por juízes independentes, selecionando aleatoriamente as unidades de análise e utilizando procedimentos estatísticos para análise de dados). Embora tais procedimentos possam ser vistos inicialmente como alheios à tradição psicanalítica, é importante salientar que a adoção de medidas empíricas nos estudos de caso de tratamentos psicodinamicamente orientados não representa uma ruptura, mas sim um acréscimo que visa enriquecer e ampliar as possibilidades de estudo.

Em síntese, os estudos de caso, qualitativos ou quantitativos, sistemáticos e intensivos são extremamente relevantes e úteis no estudo das psicoterapias psicanalíticas. Auxiliam na compreensão do processo de mudança, possibilitam geração de novas teorias e o teste das já existentes, e permitem que as psicoterapias sejam estudadas levando em consideração a dimensão subjetiva inerente ao encontro terapêutico, oferecendo assim uma alternativa para a superação da dissociação entre atividade clínica e atividade científica.

\section{Referências}

Albani, C., Pokorny, D. Blaser, G., Geyer, M., \& Kächele, H. (2007). Studying the Core Conflictual Relationship Theme (CCRT). In H. Kächele, J. Schachter, Thöma, H. \& the UIm Psychoanalytic Process Research Study Group, From
Psychoanalytic Narrative to Empirical Single Case Research. Implications for Psychoanalytic Practice (pp.288308). New York: Ulm. Retrieved January 22, 2007, from: $<$ http://sip.medizin.uni-ulm.de/abteilung/buecher/PDF/ psych_anal_2007.pdf>.

Alves-Mazzotti, A. J. (2006). Usos e abusos dos estudos de caso. Cadernos de Pesquisa, 36 (129), 637-651.

Ablon, J. S., \& Jones, E. E. (2005). On analytic process. Journal of the American Psychoanalytic Association, 53, 541-568.

Araújo, M. S., \& Wiethaeuper, D. (2003). Considerações em torno das atuais correntes predominantes da pesquisa em psicoterapia. Revista Brasileira de Psicoterapia, 5 (1), 33-52.

Aveline, M., Strauss, B., \& Stiles, W. B. (2007). Pesquisa em psicoterapia. In G. Gabbard, J.S. Beck \& J. Holmes (Orgs.), Compêndio de psicoterapia de Oxford (pp.606-623). Porto Alegre: Artmed.

Backman, C. L., \& Harris, S. R. (1999). Case studies, singlesubject research and $\mathrm{N}$ of 1 randomized trials: comparisons and contrasts. American Journal of Psysical Medicine \& Reabilitation, 78 (2), 170-76.

Bucci, W. (2007). Pesquisa sobre processo. In E. Pearson, A. M. Cooper \& G. Gabbard (Orgs.), Compêndio de psicanálise (pp.320-3336). Porto Alegre: Artmed.

Chassan, J. B. (1979). Research design in clinical psychology and psychiatry. New York: Wiley .

d'Allones, C. R. (2004). O Estudo de caso: da ilustração à convicção. In C. R. d'Allones, C. Assouly-Piquet, F. B. Slama, A. Blanchet, O. Douville, A. Giami, et al. Os procedimentos clínicos nas ciências humanas (pp.69-90). São Paulo: Casa do Psicólogo.

Denscombe, M. (2003). The good research guide for smallscale social research projects. $2^{\text {nd }}$ ed. MaidenheadPhiladelphia: Open University Press.

Drozd, J. F., \& Golfried, M. R. (1996). A Critical evaluation of the state-of-the-art in psychotherapy outcome research. Psychotherapy, 33 (2), 171-80.

Eizirik, C. L. (2006). Psicanálise e pesquisa. Revista Brasileira de Psiquiatria, 28(3), 171-2.

Eells, T. D. (2007). Generating and generalizing knowledge about psychotherapy from pragmatic case studies. Pragmatic Case Studies in Psychotherapy, 3 (1), 35-54 Retrieved January 3, 2007, from: <http://pcsp.libraries. rutgers.edu>.

Flyvbjerg, B. (2006). Five misunderstandings about casestudy research. Qualitative Inquiry, 12, 219-249.

Fonagy, P. (2004). Colhendo urtigas: o impacto mútuo da psicanálise e de outras disciplinas acadêmicas na universidade. Psicanalítica, 5 (1), 29-48.

Gabbard, G. O. Introduction. In R. A. Levy \& J. S. Ablon. (2009). Handbook of evidence-based psychodynamic psychotherapy (pp.xxv-xxxiii). New York: Human Press.

Galassi, J. P., \& Gersh, T. L. (1993). Myths, misconceptions, and missed opportunity: single-case designs and counseling psychology. Journal of Counseling Psychology, 40 (4), 525-531.

Hilliard, R. B. (1993). Single-case methodology in psychotherapy process and outcome research. Journal of Consulting and Clinical Psychology, 61 (3), 373-380. 
Howard, G. S. (1993). I think I can! I think I can! Reconsidering the place for practice methodologies in psychological research. Professional Psychology: Research and Practice, 24 (3), 237-244.

Jones, E. E. (1993). Introduction to special section: singlecase research in psychotherapy. Journal of Consulting and Clinical Psychology, 61 (3), 371-372.

Jones, E. E., Ghannam, J., Nigg, J. T., \& Dyer, F. P. (1993). A paradigm for single-case research: the times series study of a long-term psychotherapy for depression. Journal of Consulting and Clinical Psychology, 61 (3), 381-394.

Kazdin, A. E. (2001). Bridging the enormous gaps of theory with therapy research and practice. Journal of Clinical Child Psychology, 30 (1), 59-67.

Kandel, E. R. (2003). A biologia e o futuro da psicanálise: um novo referencial intelectual para a psiquiatria revisitado. Revista de Psiquiatria do Rio Grande do Sul, 25 (1), 139-165

Kächele. H., Schachter, J., \& Thomä, H. (2007). Preface. In H. Kächele, J. Schachter, H. Thöma \& the Ulm Psychoanalytic Process Research Study Group. From psychoanalytic narrative to empirical single case research; Implications for Psychoanalytic Practice (pp.7-9). New York: Ulm. Retrieved January 22, 2007, from <http://sip.medizin.uni-ulm.de/ abteilung/buecher/PDF/psych_anal_2007.pdf>.

Kächele, H. \& Thomä, H. (2007). The significance of the case history in clinical psychoanalytic research. In H. Kächele, J. Schachter, H. Thomä \& the UIm Psychoanalytic Process Research Study Group. From psychoanalytic narrative to empirical single case research:implications for psychoanalytic practice (pp.108-151). New York: Ulm. Retrivied January 22, 2007, from: <http://sip.medizin.uni-ulm.de>.

Kernberg O. (2002). Present challenges to psychoanalysis. In M. Leuzinger-Bohleber \& M. Target. Outcomes of psychoanalytic treatment: perspectives for therapists and researchers (pp.323-331). London: Whure Publishers.

Lancelle, G. (1997). El psicoanálisis y la investigación en escorzo desde una perspectiva clínica. Psicoanálisis APdeBA, 19(1-2), 119-155.

Levy, R., \& Ablon, J. S. (2000). Psychoanalytic research: progress and process. Psychoanalyst Psychologist, 20 (1), 23-25.

Luyten, P., Blatt, S. J., \& Corveleyn, J. (2006). Minding the gap between positivism and hermeneutics in psychoanalytic research. Journal of the American Psychoanalytic Association, 54 (2), 571-610.

Messer, S. B. (2007). Psychoanalytic case studies and the pragmatic case study method. Pragmatic Case Studies in Psychotherapy, 3 (4), 55-58. Retrivied January 3, 2007, from: <http://pcsp.libraries.rutgers.edu>.

Mitchell, J. C. (1983). Case and situation analysis. Sociological Review, 31 (2), 187-121.
Neufeldt, S. A., \& Nelson, M. L. (1998). Research in training clinics: a bridge between science and practice. Journal of Clinical Psychology, 54 (3), 315-327.

Patton, M. Q. (1987). How to use qualitative methods in evaluation. Newbury Park, CA: Sage.

Pole, N., Ablon, J. S., O'Connor, L., \& Weiss, J. (2002). Ideal control mastery technique correlates with change in a single case. Psychotherapy: Theory, Research, Practice, Training, 39 (1), 88-96.

Robson, C. (1993). Real world research: a resource for social sciences and practicioner-researcher. Oxford: Blackwell.

Stake, R. E. (2003). Case studies. In N. K. Denzin \& Y. S. Lincoln (Orgs.), Strategies of qualitative inquiry ( $2^{\text {nd }} \mathrm{ed}$ ). Thousand Oaks, California: Sage.

Serralta, F. B., \& Streb, L. G. (2003). Notas sobre pesquisa em psicoterapia psicanalítica: situação atual e perspectivas. Revista Brasileira de Psicoterapia, 5 (1), 53-66.

Silvares, E. (2004) Estudos de caso em psicologia clínica comportamental ( $3^{\mathrm{a}} \mathrm{ed}$.). Campinas: Papirus.

Wallerstein, R. S. (2007). Pesquisa sobre resultados. In E. Pearson, A. M. Cooper \& G. Gabbard. Compêndio de psicanálise (pp.305-319). Porto Alegre: Artmed.

Wallerstein R.S. (2002). The generations of psychotherapy research: an overview. In: M. Leuzinger-Bohleber \& M. Target. Outcomes of psychoanalytic treatment:perspectives for therapists and researchers. London: Whure Publishers.

Weiss, A. P. (2009). Measuring and Enhancing the Impact of Psychodynamic Psychotherapy Research. In R. A. Levy \& J. S. Ablon. Handbook of Evidence-Based Psychodynamic Psychotherapy (pp.389-393). New York: Human Press.

Westen, D. (2002). The languaje of psychoanalytic discourse. Psychoanalytic Dialogues, 12 (6), 857-898.

Widlöcher, D. (1994). A case is not a fact. International Journal Psycho-Analysis, 75, 1233-1244.

Yacuzzi, E. (2005). El estudio de caso como metodologia de investigación: teoría, mecanismos causales, validación. (Serie Documentos de Trabajo). Recuperado outubro 15, 2007, disponible <http://www.cema.edu.ar/ publicaciones/download/ documentos/296.pdf>.

Yin, R. K. (1981). The case crisis: some answers. Study Administrative Science Quarterly, 26 (1), 58-66.

Yin, R. K. (2001). Estudo de caso: planejamento e métodos (2a ed). Porto Alegre: Bookman.

Recebido em: 3/8/2009

Versão final reapresentada em: 7/12/2010

Aprovado em: 1/6/2011 